\title{
Reabilitação estética por meio de restauração direta em paciente pediátrico vítima de trauma dental: relato de caso
}

\author{
Aesthetic rehabilitation through direct restoration in a pediatric patient victim of dental \\ trauma: case report
}

\section{Rehabilitación estética a través de la restauración directa en paciente pediátrico víctima del trauma dental: informe de un caso}

Maria Vitoria Oliveira Dantas ${ }^{1 *}$, Amanda da Silva Araújo', Paula Lima Nogueira ${ }^{1}$, Quemuel Pereira da Silva ${ }^{1}$, Fausta Maria Moura de Castro ${ }^{1}$, Filipe de Oliveira Lima ${ }^{1}$, Estefânia Queiroga de Santana e Alencar², Elizandra Silva da Penha1, Rosana Araújo Rosendo¹, Luanna Abílio Diniz Melquiades de Medeiros ${ }^{1}$.

\section{RESUMO}

Objetivo: Apresentar um relato de caso, onde foi proposto reabilitar o sorriso de um paciente pediátrico vítima de trauma dental, por meio de restauração direta em resina composta. Detalhamento do caso: Paciente A.S.M., melanoderma, sexo masculino, 9 anos de idade, apresentou-se à Clínica Escola relatando queda da própria altura e nessa o acometimento dos elementos 11 e 21 . O paciente apresentou-se após 6 meses do trauma mencionando como motivo principal da sua consulta o bullying. Durante a anamnese foi informado que imediatamente após o trauma houve ausência de injúria aos tecidos moles, desmaio ou sangramento nasal. Posteriormente, o exame clínico e radiográfico foi realizado e através deles foi diagnosticada uma fratura coronária não complicada, esta por sua vez, atingindo apenas esmalte e dentina, não havendo envolvimento pulpar e periodontal. A reabilitação estética e funcional foi realizada através de restauração classe IV nos elementos supracitados. Considerações finais: $O$ atendimento realizado mostrou-se eficaz na reabilitação do sistema estomatognático, tanto considerando a função como a estética, levando em conta todas as peculiaridades de um atendimento odontopediátrico. Assim como impactou positivamente a autoestima da criança, devolvendo para ele a espontaneidade do seu sorriso através de uma alternativa terapêutica conservadora.

Palavras-chave: Estética dentária, Odontopediatria, Traumatismos dentários.

\section{ABSTRACT}

Objective: To present a case report, in which it was proposed to rehabilitate the smile of a pediatric patient victim of dental trauma, by means of direct restoration in composite resin. Case details: Patient ASM, melanoderma, male, 9 years old, presented to Clínica Escola reporting a fall from his own height and in this case the involvement of elements 11 and 21. The patient presented after 6 months of trauma mentioning as the main reason for his consults bullying. During the anamnesis, it was reported what immediately after the trauma, there was absence soft tissue injury, fainting or nasal bleeding. Posteriorly, the clinical and radiographic examination was performed and through them uncomplicated coronary fracture was diagnosed, are in turn, affecting only enamel and dentin, with no pulp and periodontal involvement. The aesthetic and functional rehabilitation was carried out through class IV restoration in the aforementioned elements. Final considerations: The care provided showed to be effective in the rehabilitation of the stomatognathic system, both considering function and aesthetics, taking into account all the peculiarities of pediatric dental care. As well as positively impacting the child's self-esteem, returning to him the spontaneity of his smile through a conservative therapeutic alternative.

Key words: Esthetics dental, Pediatric dentistry, Tooth injuries.

1Universidade Federal de Campina Grande (UFCG), Patos - PB. *E-mail: mvitoria.od@hotmail.com ${ }^{2}$ Centro Universitário de Patos (UNIFIP), Patos - PB. 


\section{RESUMEN}

Objetivo: Presentar un informe de caso, en el que se propuso rehabilitar la sonrisa de un paciente pediátrico que sufre un trauma dental, mediante restauración directa en resina compuesta. Detalles del caso: Paciente ASM, melanoderma, masculino, de 9 años, se presentó a la Clínica Escola informando caer desde su propia altura y en esto la participación de los elementos 11 y 21 . El paciente presentó después de 6 meses del trauma mencionando como la razón principal de su consulta el bullying. Durante la anamnesis, se informó que inmediatamente después del trauma, no hubo lesiones de tejidos blandos, desmayos o sangrado nasal. Posteriormente, se realizó el examen clínico y radiográfico y, a través de ellos, se diagnosticó una fractura coronaria no complicada, que a su vez solo afectaba el esmalte y la dentina, sin afectación pulpar y periodontal. La rehabilitación estética y funcional se llevó a cabo a través de la restauración clase IV en los elementos antes mencionados. Consideraciones finales: La atención brindada demostró ser efectiva en la rehabilitación del sistema estomatognático, considerando la función y la estética, teniendo en cuenta todas las peculiaridades de la atención pediátrica. Además de impactar positivamente la autoestima del niño, devolviéndo la espontaneidad de su sonrisa a través de una alternativa terapéutica conservadora.

Palabras clave: Estética dental, Odontología pediátrica, Traumatismos de los dientes.

\section{INTRODUÇÃO}

O traumatismo dentário é uma urgência recorrente no atendimento odontopediátrico que requer inúmeros cuidados especiais, uma vez que causa abalo psicológico não somente ao paciente, como também aos responsáveis e familiares envolvidos. Esses atendimentos são desafiadores para o cirurgião dentista, pois muitas vezes requerem um arsenal de técnicas para o manejo do comportamento; visto que esses pacientes comumente apresentam sintomatologia dolorosa, estado de sofrimento e ansiedade que podem ser agravados por procedimentos mais invasivos e que geram um maior grau de estresse e resistência (TEIXEIRA BCS, et al., 2019; AVELAR PN, 2019).

A maioria das lesões dentárias ocorrem por brincadeiras, prática de esportes, quedas da própria altura, acidentes em parques recreativos e acidentes automobilísticos; e variam de acordo com o sexo (SANTOS $\mathrm{KS}$, et al., 2016). O tratamento destas é muitas vezes negligenciado, embora essas lesões possam causar dor, perturbações na fala e mastigação, assim como implicar na autoestima da criança (MUHAMAD AH, et al., 2019a).

Os traumatismos são classificados naqueles que acometem os tecidos duros dentais e naqueles que acometem os tecidos de suporte. As fraturas coronárias que envolvem esmalte e dentina de incisivos centrais superiores ocorrem na maioria dos casos, como também é frequente em crianças, ocorrendo em 19,4 a 49\% dos pacientes. O tratamento depende do tipo de trauma, condição pulpar e periodontal e do estágio de desenvolvimento radicular (MUHAMAD AH, et al., 2019b; PRADO MC, et al., 2018).

As fraturas coronárias descomplicadas referem-se a perda da estrutura dental de esmalte e dentina, sem o comprometimento pulpar. Clinicamente possui mobilidade normal, teste de vitalidade pulpar positivo, ausência de dor a percussão e palpação, e pode apresentar sensibilidade dentária. A terapêutica pode consistir na colagem do fragmento dental, mas quando não é possível, a restauração direta em resina composta é a abordagem mais indicada (BARROS IRV, et al., 2020).

Por ser a opção considerada mais conservadora, minimamente invasiva, com pouco ou nulo desgaste dental, melhor custo-benefício e com excelente longevidade, a restauração em resina composta pela técnica direta atinge todos os objetivos desejados e é bem sucedida em dentes jovens (MUHAMAD AH, et al., 2019a). Diante dos avanços dos materiais restauradores, as resinas compostas dispõem de maior facilidade de manipulação e da mimetização das características da estrutura dental, permitindo uma estética natural aos dentes (DIEGUES MA, et al., 2017).

Posto isso, o objetivo do presente artigo é descrever e discutir um caso clínico no qual foi efetuado uma intervenção estética e funcional por meio de restauração direta em resina composta, avaliando as características clínicas e radiográficas, com o propósito de reabilitar o sorriso de um paciente pediátrico vítima de trauma dental. 


\section{DETALHAMENTO DO CASO}

Paciente A.S.M., melanoderma, sexo masculino, 9 anos de idade, apresentou-se à Clínica Escola de Odontologia da Universidade Federal de Campina Grande - UFCG, campus Patos/PB, relatando a ocorrência de um trauma na região de dentes anteriores superiores, há mais de 6 meses. Durante anamnese, foi relatada fratura por trauma após uma queda da própria altura enquanto brincava na escola e ao exame clínico observou-se fraturas do tipo classe IV nos elementos 11 e 21 devido ao trauma provocado, atingindo apenas esmalte e dentina, sem envolvimentos pulpar ou periodontal, sendo então classificadas como fraturas coronárias não complicadas (Figura 1).

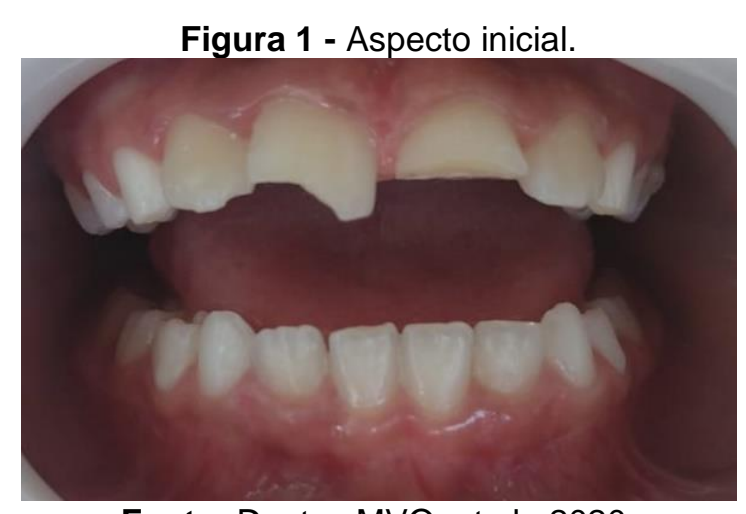

Fonte: Dantas MVO, et al., 2020.

Foi relatado no momento pós trauma, ausência de injúria aos tecidos moles, desmaio ou sangramento nasal. Ainda na anamnese foi relatada uma certa mudança de comportamento do paciente após as fraturas, pois o mesmo passou a sofrer bullying por parte dos colegas de escola. Neste momento da primeira consulta foi realizada profilaxia e radiografia periapical dos elementos (Figura 2).

Figura 2 - Radiografia periapical dos elementos.

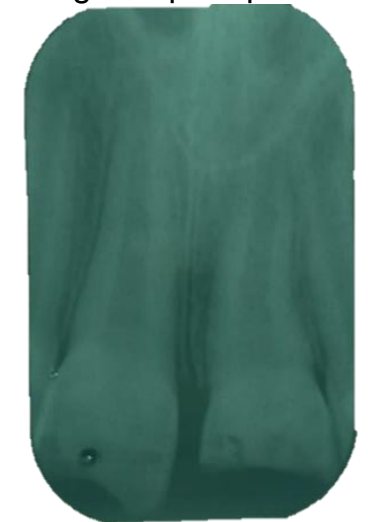

Fonte: Dantas MVO, et al., 2020.

Radiograficamente, o tecido pulpar e o periodonto se apresentavam dentro dos padrões de normalidade. Buscando direcionar o diagnóstico, foi realizado teste de vitalidade pulpar ao frio, que respondeu também dentro dos padrões de normalidade.

Após avaliação minuciosa, optou-se pela realização de um tratamento restaurador direto com resina composta. A primeira etapa do procedimento restaurador foi a realização de uma simulação estética para definir a escolha da cor, a partir da colocação de incrementos de resina sobre o dente a ser restaurado não condicionado, e sem uso do sistema adesivo. Ficando estabelecidas as seguintes cores de resina: A2B e A2E da resina Z350® (3M ESPE - São Paulo, SP) e universal da resina Z100® (3M ESPE - São Paulo, SP). Após seleção da cor, iniciou-se o preparo conservador dos elementos com ponta diamantada 2135, que constituiu apenas na confecção do bisel, a fim de melhorar a estética da interface dente/restauração e aumentar a área de adesão (Figura 3). 
Figura 3 - Confecção do bisel com ponta diamantada 2135.

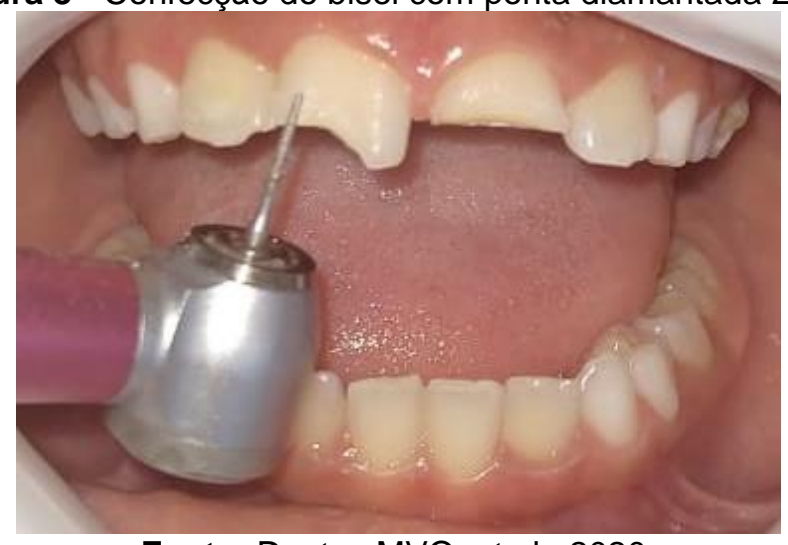

Fonte: Dantas MVO, et al., 2020.

A partir disto, sob isolamento relativo modificado, ou seja, usando-se o afastador labial, roletes de algodão e sugador de saliva, foi realizado condicionamento com ácido fosfórico a 37\% (FGM - Joinville, SC) por 30 segundos (Figura 4), o local foi lavado abundantemente com água destilada e seco com leve jato de ar.

Figura 4 - Condicionamento com ácido fosfórico a 37\%.

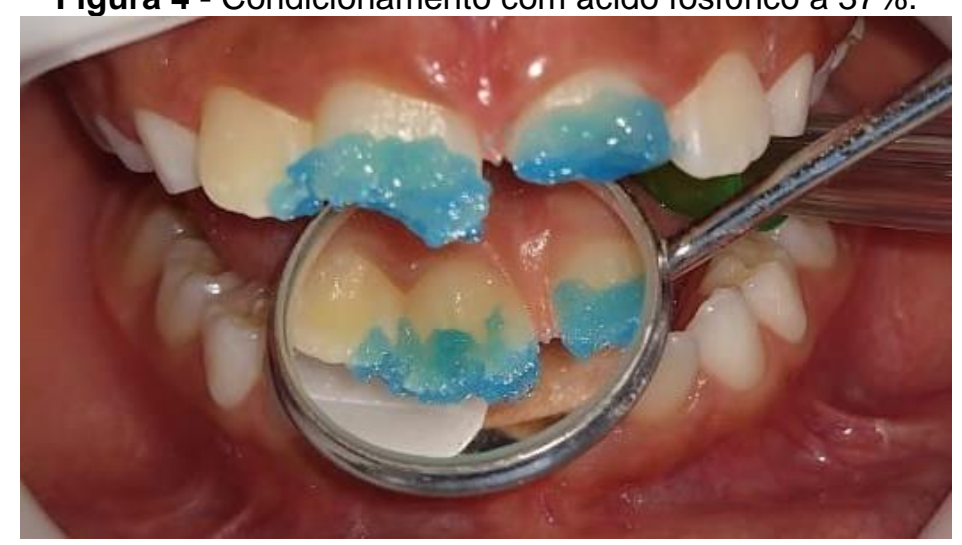

Fonte: Dantas MVO, et al., 2020.

Com o auxílio de microbrush (3M/ESPE) foi aplicado o sistema adesivo Adper Single Bond® (3M ESPE Sumaré, SP) e foto polimerizado durante 40 segundos. Em seguida, aplicou-se com auxílio de uma espátula de titânio, incrementos de resina A2B (Z350) nas áreas correspondentes à dentina perdida (Figura 5). Utilizou-se a resina A2E (Z350) para as camadas referentes ao esmalte e por fim resina composta universal (Z100) para confecção das bordas incisais. Para a confecção da parede palatina foi utilizada uma tira de poliéster.

Figura 5 - Aplicação de incrementos de resina.

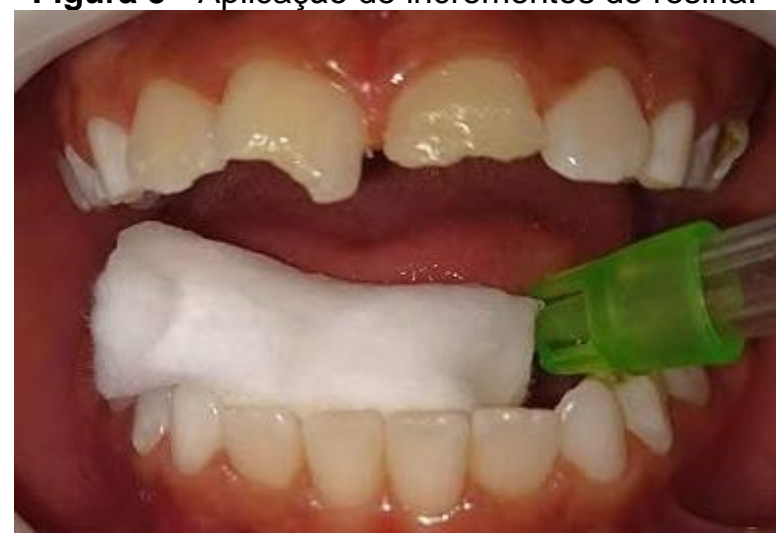

Fonte: Dantas MVO, et al., 2020. 
Após finalização da restauração, em outra sessão foram realizados acabamento e polimento final. Foram utilizadas ponta diamantada $2135 \mathrm{FF}$, tiras de lixa, discos de lixa e borrachas siliconadas (3M ESPE - São Paulo, SP). Enfim, foi utilizada roda de feltro embebida com pasta abrasiva, obtendo-se uma superfície brilhante e lisa nas faces vestibulares (Figura 6A) e nas faces palatinas (Figura 6B) resultante do polimento. O responsável pelo paciente foi orientado quanto a necessidade de proservação do caso e retorno para avaliação radiográfica das condições pulpar e periodontal.

Figura 6 - Vista vestibular do resultado final (A), vista palatina após realização de acabamento e polimento (B).
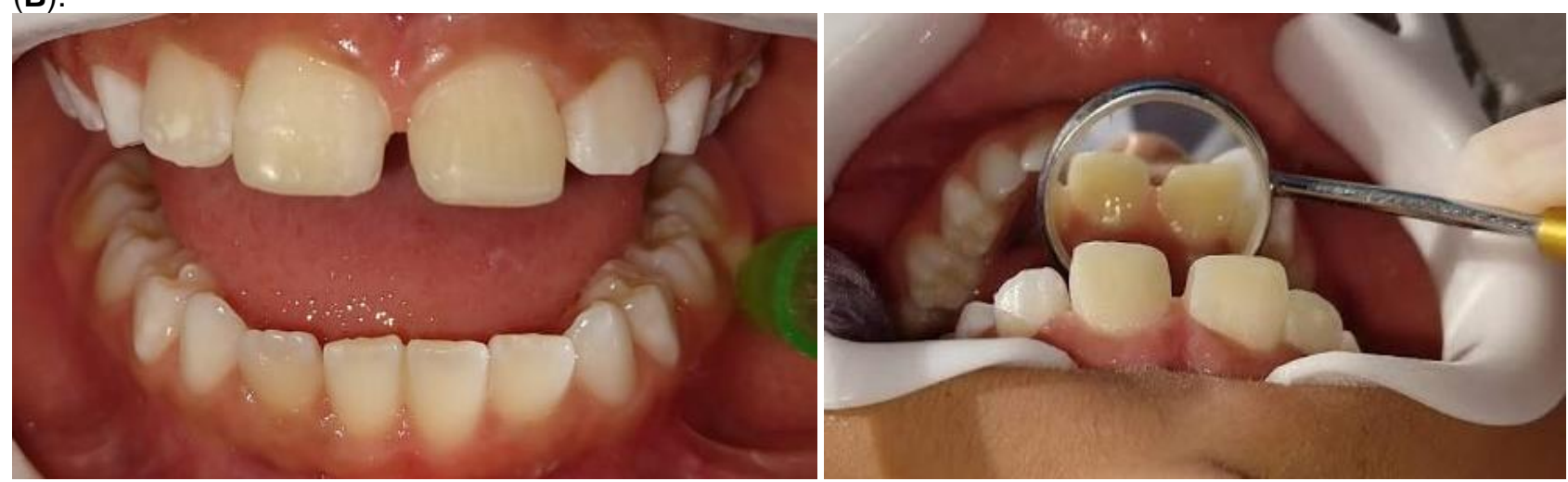

Fonte: Dantas MVO, et al., 2020.

\section{DISCUSSÃO}

O trauma dental é compreendido como uma agressão na qual a sua potência supera a resistência presente nos tecidos duros ósseos e dentários, podendo ser térmica, química ou mecânica. $\mathrm{O}$ tipo, a duração e a intensidade do impacto definem a extensão da lesão. Frente ao declínio dos índices de cárie dentária no Brasil, o traumatismo dentário tem se destacado como um crescente problema de saúde pública e se tornou alvo de pesquisas científicas, principalmente na odontopediatria (COSTA SC, et al., 2019).

Estudos epidemiológicos mostram que a cada duas crianças, uma sofre traumatismo dentário, a idade mais acometida está entre 7 e 12 anos, predominando o sexo masculino (FERREIRA S e GUEDES-PINTO AC, 2016), e no Brasil, $20 \%$ a $31 \%$ dos escolares são vítimas desse incidente. Segundo pesquisas, os incisivos centrais superiores são os elementos mais acometidos, e um terço desses dentes apresentam rizogênese incompleta (ARECO IM, 2017; MOTA LQ, et al., 2015; KINA M, et al., 2015). Perfil no qual o paciente do presente relato participa, possuindo 9 anos de idade, sexo masculino, escolar e apresentando fratura dental nos elementos 11 e 21.

O atendimento de urgência de traumas é primordial para o sucesso do tratamento, porém, devido ao despreparo da população e dos profissionais de saúde, esse atendimento tem sido inadequado ou até mesmo negligenciado. Em se tratando dos fatores etiológicos, esportes e jogos com bolas geralmente aparecem como um dos fatores principais para crianças de idade escolar, diante disso, pesquisas mostram que cuidadores e professores carecem de conhecimento apropriado para lidar com crianças vítimas de trauma dental (PRESTES CAB, et al., 2019; TAKAHASHI K, et al, 2019).

O traumatismo dentário pode produzir fraturas coronárias e corono-radiculares, fraturas radiculares, concussão, subluxações, luxações, avulsões e fraturas alveolares, como também lesionar os tecidos moles. O plano de tratamento procede da extensão da lesão, condição endodôntica e periodontal, e do estágio de desenvolvimento radicular, assim como podem demandar procedimentos de dentística, endodontia, prótese, periodontia, ortodontia e cirurgia (FIGUEIREDO LR, et al., 2017).

As lesões causadas aos tecidos moles e de suporte dos dentes podem resultar em sangramentos, gerando medo a criança e aos acompanhantes, que consequentemente levam a rápida procura por atendimento. De outro modo, as lesões podem ocorrer com a ausência de sangramentos e sintomas, que por sua vez acarreta a não procura por atendimento (VIANA KAS, et al., 2019), circunstância que ocorreu no caso em questão, onde $\mathrm{o}$ atendimento em decorrência do trauma dental só se realizou após 6 meses. 
Perante a negligência do atendimento odontológico imediatamente após o trauma dental, inúmeras complicações podem discorrer, como dor, dificuldade na fala e na mastigação, bem como as alterações no sorriso da criança podem provocar bullying. A literatura revela que as características físicas mais comuns que causam provocações entre as crianças são os dentes, cabelos, peso e altura (BATISTA JFNF, et al, 2019). Apesar das disfunções, o bullying foi a principal queixa relatada pela criança e por seu responsável, onde mencionaram episódios ocorridos na escola.

Tratamentos de urgência odontológica possuem caráter invasivo e causam efeitos biológicos e emocionais para os pacientes, Avelar PN (2019), relata que para os atendimentos de urgência, as crianças são 50\% menos colaboradoras e duas vezes mais opositoras quando comparadas aos procedimentos simples como por exemplo a profilaxia. Posto isso, entende-se a necessidade do domínio das técnicas operatórias, como também das técnicas de condicionamento do manejo do comportamento de pacientes infantis.

O paciente em questão relatou ausência de sintomatologia dolorosa e ao exame radiográfico e de vitalidade pulpar constatou-se o não envolvimento pulpar e dos tecidos periapicais. Diante do primeiro atendimento odontológico do paciente em questão, o plano de tratamento consistiu na opção considerada a mais conservadora e que atinge os objetivos estéticos e funcionais para o paciente. Assim, para corroborar com a técnica, optou-se por um protocolo menos invasivo, empregando o isolamento relativo modificado quando a criança apresentou resistência ao isolamento absoluto do campo operatório (SANTOS FG, et al., 2016).

Fraturas de coroa que envolvem dentina e esmalte, sem o envolvimento pulpar, são classificadas como fraturas não complicadas, e acometem frequentemente dentes anteriores permanentes de crianças e adolescentes. A reabilitação estética é um desafio e exige do dentista habilidades, domínio da técnica restauradora e conhecimento da morfologia dental a ser reproduzida (MUHAMAD AH, et al., 2016). A restauração direta em resina composta é uma técnica minimamente invasiva, econômica e bem-sucedida para restaurações em fraturas dentárias, além de apresentar estética natural e excelente longevidade (MUHAMAD AH, et al., 2019a).

Com o passar dos anos tornou-se evidente a evolução da qualidade dos materiais dentários restauradores, havendo uma melhora considerável na mimetização da cor, para uma estética natural, e na facilidade de manipulação. Através da técnica de restauração direta, a resina composta é aplicada diretamente sobre o dente, em pequenos incrementos, por isso, o protocolo clínico deve ser seguido criteriosamente para o êxito do resultado final (DANTAS MVO, et al., 2020). Etapas precedentes como seleção do material, seleção da cor, isolamento do campo operatório efetivo e a confecção do bisel para mascarar a interface denterestauração, são importantes e foram respeitadas durante todo o protocolo do presente caso.

Mesmo 6 meses após o trauma dental, o paciente apresentou comportamento tenso, justificado pelo primeiro contato da criança com a clínica odontológica, entretanto, apesar da tensão, o paciente mostrou-se cooperador durante todo o procedimento clínico. Diante das extensas fraturas e do acometimento psicológico causado a criança pelo bullying, a reabilitação estética foi categorizada como urgência, necessitando de um protocolo restaurador menos invasivo e de curta duração, visando reduzir a exacerbação de estímulos estressantes para manter o comportamento cooperativo da criança.

Diante da adversidade do atendimento de urgência, o manejo do comportamento e dos anseios do paciente pediátrico frente a fratura dental é desafiador. A elaboração do plano de tratamento e o domínio das técnicas restauradoras são fundamentais para o sucesso estético e funcional da reabilitação do sorriso harmônico da criança. Os responsáveis pela criança foram informados sobre a necessidade de acompanhamento clínico e radiográfico dos elementos 11 e 21. A vista disso, torna-se necessário a realização de mais pesquisas sobre a abordagem terapêutica de pacientes pediátricos vítimas de trauma dental, assim como das técnicas restauradoras para a resolução de fraturas coronárias descomplicadas e sua proservação.

Por meio desse relato de caso, foi possível discorrer uma abordagem terapêutica de um paciente infantil vítima de trauma dental que compareceu à Clínica Escola de Odontologia da UFCG. O atendimento clínico resultou de forma eficaz na resolução da estética e função do sistema estomatognático, assim como impactou de forma positiva a autoestima da criança e confirmou uma alternativa terapêutica conservadora e rápida da técnica de restauração direta em resina composta. 


\section{REFERÊNCIAS}

1. ARECO IM. Considerações atuais de intrusão dentária: Revisão de literatura. Monografia (Especialista em Endodontia). Faculdade de odontologia de Piracicaba. Universidade estadual de Campinas, 2017.

2. AVELAR PN. Procedimentos de urgência em odontopediatria: dificuldades do atendimento no serviço de pronto socorro odontológico de Uberlândia. Trabalho de Conclusão de Curso (Graduação em Odontologia) - Universidade Federal de Uberlândia, 2019; 1-29.

3. BARROS ÍRV, et al. Traumatismos dentários: da etiologia ao prognóstico, tudo que o dentista precisa saber. Revista Eletrônica Acervo Saúde, 2020; (45): e3187-e3187.

4. BATISTA JFNF, et al. Intrusão de elemento dentário decorrente de trauma em paciente infantil: relato de caso. Trabalho de Conclusão de Curso (Graduação em Odontologia). Universidade Tiradentes, 2019; 1-23.

5. COSTA SC, et al. Terapia Preventiva Pós Traumatismo Dental na Primeira Infância por Reabilitação Protética Funcional: Relato de Caso. Revista Eletrônica Acervo Saúde, 2019; (26): e766-e766.

6. DANTAS MVO, et al. Fechamento de diastema com auxílio de guia palatino: relato de caso. Research, Society and Development, 2020; 9(7): 1-17.

7. DIEGUES MA, et al. Cerâmica x resina composta: o que utilizar? Revista Uningá, 2018; 51(1): 87-94.

8. FERREIRA S, GUEDES-PINTO AC. Manejo da criança no consultório. Odontopediatria, 9a ed. São Paulo: Santos, 2016.

9. FIGUEIREDO LR, et al. DExt 011-Impacto na formação do estudante e na transformação social com a reabilitação integral do indivíduo após o traumatismo dentoalveolar. Archives Of Health Investigation, 2017; 6.

10. KINA M, et al. Dente anterior fraturado: diagnóstico, prognóstico e retratamento de caso clínico. Archives Of Health Investigation, 2015; 4(1): 20-25.

11. MOTA LQ, et al. Luxação Intrusiva e Fratura Dental com Inserção do Fragmento no Lábio Inferior: Relato de Caso. Journal of Health Sciences, 2015; 16(2): 129-33.

12. MUHAMAD AH, et al. Anterior Esthetic Restorations Using Direct Composite Restoration; a Case Report. Dentistry \& Dent Pract J, 2019b; 2(1): 1-6.

13. MUHAMAD AH, et al. Restoring Fractured Anterior Tooth Using Direct Composite Restoration: A Case Report. Global Journal of Dental Sciences, 2019a; 1(1): 1-5.

14. MUHAMAD AH., et al. Prevalence of Traumatic Dental Injury in Arab Israeli Community. Journal of Dental and Medical Sciences, 2016; 15(7): 91-98.

15. PRADO MC, et al. Recurrence of dental trauma and management of pulp revascularized tooth: a case report. J Dent Health Oral Disord Ther, 2018; 9(4): 304-308.

16. PRESTES CAB, et al. Avaliação do conhecimento da abordagem do trauma dental por educadores escolares: revisão da literatura. Trabalho de Conclusão de Curso (Graduação em Odontologia). Centro Universitário de Anápolis. UniEvangélica, 2019; 1-41.

17. SANTOS FG, et al. Reabilitação Estética em Dentes Anteriores Permanentes Traumatizados. Journal of Health Sciences, 2016; 18(3): 195-200.

18. SANTOS KS, et al. Fratura radicular horizontal no terço apical: relato de caso. RGO-Revista Gaúcha de Odontologia, 2016; 64(4): 467-471.

19. TAKAHASHI K, et al. Traumatic dental injuries in young children. Archives Of Health Investigation, $2019 ; 8$ (3): 113118.

20. TEIXEIRA BCS, et al. Abordagem terapêutica de fratura radicular com 30 meses de acompanhamento. Relato de caso clínico. Revista Odontológica do Brasil Central, 2019; 28(85): 82-86.

21. VIANA KAS, et al. Traumatismo dentário na dentição decídua. Anais do Seminário Científico do UNIFACIG, 2019; (5): $1-10$. 